\title{
CHARACTERIZATION OF AN ENHANCER ELEMENT THAT REGULATES FOXP2 EXPRESSION IN HUMAN CEREBELLUM
}

\author{
Julen Madurga and Francisco J. Novo (correspondence: fnovo@unav.es) \\ Department of Biochemistry and Genetics. School of Science. University of Navarra, 31008 Pamplona (Spain).
}

\begin{abstract}
Human cerebellum is involved not only in motor control but also in several cognitive skills. In this work, our goal was to identify genomic enhancers responsible for the regulation of genes implicated in the development of this brain region. From an initial collection of Vista elements showing specific enhancer activity in mouse hindbrain, we found an enhancer element located within the final intron of FOXP2, a gene expressed in cerebellum and implicated in vocal communication and speech and language disorders in humans. Analysis of this enhancer using various computational resources suggests that it is a strong candidate to account for FOXP2 expression during cerebellar development. Two blocks within this region are deeply conserved in vertebrates; they are separated by a microsatellite sequence present only in eutherian mammals, and expanded in human genomes.
\end{abstract}

\section{Introduction}

The cerebellum is a good model for the study of neurogenesis, neurodevelopment and brain evolution (1). It contains about $80 \%$ of the neurons of the CNS, even though it only represents $10 \%$ of its total mass (2). As well as its involvement in motor function, the cerebellum also participates in cognition, and shows a high degree of connectivity with the neocortex (3). In keeping with its involvement in cognitive functions, the cerebellum has expanded in hominid evolution, even when comparing archaic and modern humans (3). Likewise, cerebellar syndromes in humans frequently display some type of cognitive impairment.

As reviewed by Carroll (4), the suggestion by King and Wilson that phenotypic differences between human and chimpanzee would mostly result from regulatory changes has been subsequently confirmed; numerous findings have shown changes in cis-regulatory elements during human evolution, of which those affecting brain development are particularly relevant. FOXP2 is a good example of how changes in regulatory elements could have altered gene expression patterns during brain development and thus drive the evolution of cognitive abilities such as vocal learning. Recent research has shown the importance of transcriptomic changes during brain development in primate evolution $(5,6)$, and the role of enhancers in orchestrating such changes: many enhancers regulating genes involved in brain development harbour human-specific variants, which are frequently associated with neurodevelopmental problems (7).

As the role of enhancers in cerebellum evolution has not been extensively studied, in this work we have used several computational tools with a view to identify enhancers that are active in this brain region and regulate genes expressed during development. We have found a previously described enhancer element for which chromatin marks, gene expression profiles and chromatin contact data collectively suggest a role in FOXP2 regulation during cerebellar development.

\section{Methods}

We used several datasets available as tracks or track hubs at the UCSC Genome Browser. In order to identify and analyse enhancer regions, we took annotations from Vista enhancers (8) and GeneHancer (9). Enhancer activity in neural tissues was predicted using annotations from IDEAS $(10,11)$ to visualize chromatin states (derived from chromatin marks) in neural tissues. Transcription factor binding sites (TFBS) from ChIP-seq data were obtained from Remap (12), which includes 31 million ChIP-seq peaks from ENCODE and 49 million peaks from publicly available data.

Gene expression-GTEx RNA seq (13) was used to analyse gene expression in adult tissues. BrainSpan data (14) for specific genes were viewed in GenTree (15); the Human Developmental Biology Resource (16) was accessed via Expression Atlas (17). Both resources were used to analyse gene expression in neural tissues during various developmental stages.

GTEx Tissue eQTL (18), ClinVar Short Variants (19) and GWAS Catalog (20) were inspected to search for association between gene expression or neurodevelopmental phenotypes with specific variants located inside enhancers.

Enrichr (21,22) was used for enrichment analysis of biological categories (gene ontologies, biological pathways and expression levels in different tissues) in gene lists.

Physical interactions between candidate regulatory elements and surrounding gene promoters were 
assessed as virtual 4C plots either in the 3D Genome Browser (23) or in 3DIV (24), comparing virtual 4C plots in several neural and non-neural tissues.

The conservation track in the UCSC Genome Browser showed Multiz alignments in selected vertebrate genomes. Further alignments of candidate regulatory regions with genomic sequences were performed with CoGe Blast (25). Each candidate regulatory element was inspected in detail, selecting the core region more likely responsible for enhancer activity (PhastCons score, epigenetic marks and presence of TFBS) and used this as query in blastn searches against the genomes of species representing a range of vertebrate taxons. Table 1 lists the genome assemblies that were used in these searches.

\begin{tabular}{ll}
\hline Species & Genome assembly \\
\hline Homo sapiens & vGRCh37 (id 29349) \\
Pan troglodytes & v2.1.4 (id28817) \\
Otolemur garnettii & v3 (id 16664) \\
Mus musculus & vGRCm38.p5 (id 34107) \\
Bos taurus & v3.1 (id 34644) \\
Loxodonta africana & v3 (id 16685) \\
Monodelphis domestica & v5 (id 35487) \\
Ornithorhynchus anatinus & v5.0.1 (id 28045) \\
Gallus gallus & v4 (id 28417) \\
Xenopus tropicalis & v9.1 (id 52485) \\
Latimeria chalumnae & v1.0 (id25005) \\
Danio rerio & v10 (id 25001)0 \\
Gasterosteus aculeatus & v2 (id 52353) \\
Takifugu rubripes & v5 (id 25444) \\
Tetraodon nigroviridis & vUCSC (id23254) \\
Lepisosteus oculatus & v1 (id 52331) \\
Callorhinchus milii & v6.1.3 (id 25133) \\
Eptatretus burgeri & v3.2 (id 52454 \\
Petromyzon marinus & v7.0.64 (id 12390) \\
\hline
\end{tabular}

Table 1. Genome assemblies used in COGE blastn searches.
Enrichr. As shown in Table 2, this gene list is significantly over-represented ( $p_{\text {adjusted }}<0.05$ ) in genes upregulated in several brain regions according to ARCHS ${ }^{4}$ Tissues database (26), cerebellum showing the highest absolute Z-score value (1.69). A similar result was obtained in the Cancer cell line Encyclopedia database, with a significant enrichment in genes expressed in neural cell lines $\left(\mathrm{p}_{\text {adjusted }}=2.1 \times 10^{-4}\right)$; in the Tissue Protein Expression from Human Proteome Map database, significant enrichment was found exclusively in fetal brain, ( $p_{\text {adjusted }}=0.014$ ), and in Jensen Tissues database (27) the highest absolute Z-score (4.12) corresponded to ectoderm ( $\mathrm{p}_{\text {adjusted }}=5.3 \times 10^{-4}$ ).

\begin{tabular}{lrrrr} 
Name & \multicolumn{1}{c}{ P-value } & Adjusted p-value & \multicolumn{1}{c}{ Z-score } & Combined score \\
\hline NEURONAL EPITHELIUM & $3.508 \mathrm{e}-7$ & 0.00003789 & -1.58 & 23.52 \\
PREFRONTAL CORTEX & 0.00001297 & 0.0007006 & -1.54 & 17.36 \\
CEREBELLUM & 0.00003909 & 0.001055 & -1.69 & 17.13 \\
MOTOR NEURON & 0.00003909 & 0.001055 & -1.64 & 16.66 \\
SPINAL CORD & 0.0001118 & 0.002013 & -1.62 & 14.75 \\
SPINAL CORD (BULK) & 0.0001118 & 0.002013 & -1.59 & 14.46 \\
MIDBRAIN & 0.0007797 & 0.009357 & -1.46 & 10.45 \\
RENAL CORTEX & 0.0007797 & 0.009357 & -1.43 & 10.23 \\
FETAL BRAIN CORTEX & 0.0007797 & 0.009357 & -1.39 & 9.97 \\
FETAL BRAIN & 0.001897 & 0.01707 & -1.47 & 9.23
\end{tabular}

Table 2. Enrichment analysis of 93 genes located near Vista elements active in hindrain. Genes in this list are enriched in genes upregulated in brain regions, according to $A R C H S^{4}$ Tissues database. Only significant values (adjusted p-value) are shown. Z-scores indicate, for every category, the variation in rank (standard deviations) in the gene list compared to the background list.

These results support the assumption that these Vista enhancers collectively tend to regulate genes involved in neurodevelopmental processes. In order to identify which of these enhancers might be associated with cerebellum, we used a combination of approaches: (i) presence of eQTLs or variants associated with a neurodevelopmental phenotype (by GWAS or in ClinVar); (ii) expression pattern of the genes surrounding each Vista element should be consistent with its involvement in cerebellar function, and (iii) presence of epigenetic marks of active enhancer in neural tissues. This led to a short list of three Vista enhancers very likely to be involved in cerebellar function and/or development, shown in Table 3.

\section{Results}

We searched for all VISTA elements that show activity in mouse hindbrain, either exclusively or in hindbrain and neural tube. This resulted in a set of 58 enhancers $(38$ active exclusively in hindbrain and 20

\begin{tabular}{lllll}
\hline Enhancer & GRCh37 coordinates & Active & $\begin{array}{l}\text { Epigenetic marks of active } \\
\text { enhancer in: }\end{array}$ & $\begin{array}{l}\text { Overlaps } \\
\text { with: }\end{array}$ \\
\hline hs966 & chr7:114326912-114329772 & $r$ & Neural (embryonic and adult) & FOXP2 \\
hs1082 & chr11:31816452-31818421 & $r+n t$ & Neural (embryonic) & PAX6 \\
hs2094 & chr1:10795106-10799241 & $r$ & Neural and non-neural & CASZ1
\end{tabular}
active in hindbrain and neural tube). In order to confirm the reliability of this dataset, we made a list with all genes flanking these enhancers up to a distance of $1 \mathrm{Mb}$ in each direction (93 genes) and analysed the enrichment of several biological categories using
Table 3. VISTA enhancers selected after initial analysis. " $r$ " indicates that the enhancer is active in rhombencephalon, "nt" in neural tube. Genome coordinates in GRCh37 assembly. 
bioRxiv preprint doi: https://doi.org/10.1101/641597; this version posted May 17, 2019. The copyright holder for this preprint (which was not certified by peer review) is the author/funder, who has granted bioRxiv a license to display the preprint in perpetuity. It is made available under aCC-BY-NC-ND 4.0 International license.

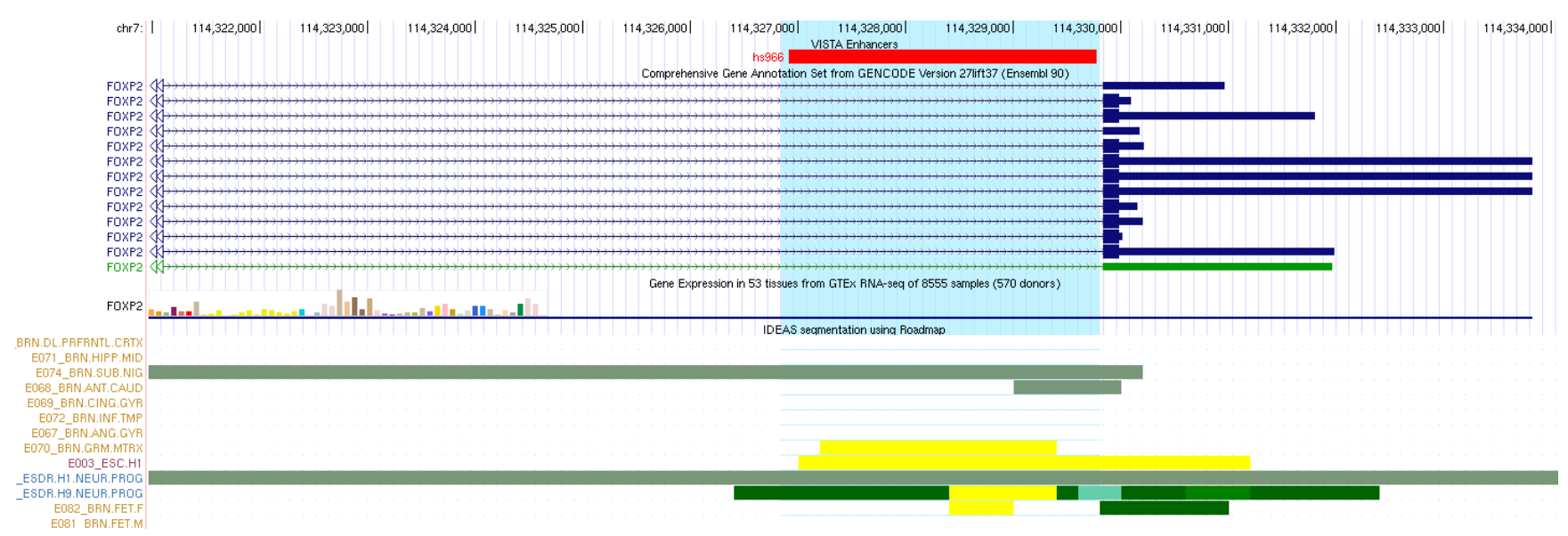

Figure 1. Location and chromatin marks of Vista element hs966 (in red at the top). Rectangles in the bottom tracks indicate active enhancer status (yellow) or transcription (green) according to the IDEAS Roadmap chromatin segmentation method.

The hs966 Vista element is located in the final intron of FOXP2, shows high and specific activity in mouse hindbrain according to Vista and bears marks of active enhancer in several brain samples and progenitor cell lines from the Roadmap Epigenetics Project (Figure 1): germinal matrix (E070), male fetal brain (E081), female fetal brain (E082), H1 ESCs (E003) and H9 derived neuronal progenitor cells (E009).

As all Vista elements, hs966 is included in GeneHancer database (enhancer GH07J114686), which predicts its association with FOXP2 only on the grounds of its distance to the promoter of this gene (no evidence based on eQTLs, capture HiC, eRNA co-expression or transcription factor co-expression). Given the prior association of FOXP2 with vocal communication through Purkinje cell development and with speech and language disorders (28-30) we decided to search for further evidence that the hs966 element constitutes a good candidate to regulate FOXP2 expression in cerebellum during development.

First, we searched for data on FOXP2 expression throughout human development. Although expression in adult brain tissues is not particularly high according to GTEx, data from Brainspan show a clear peak of FOXP2 expression in cerebellum, and to a lesser extent in midbrain, around post-conception week (pcw) 12 of human embryonic development (Figure 2). Expression goes down quickly and is then maintained at lower levels until 32 pcw when it decreases to postnatal levels. A lower expression burst is also evident in embryonic striatum, again disappearing in late-prenatal and postnatal samples.
Next, we searched for HiC datasets from brain samples and compared them with non-neural tissues, in an effort to find further experimental evidence for the contact between this enhancer and the promoter of FOXP2. Although, to the best of our knowledge, $\mathrm{HiC}$ experiments are not available in human developing cerebellum or Purkinje cells, we explored contacts in datasets from $\mathrm{H} 1$ neuronal progenitor cells (GSM1267202; GSM1267203), cerebellar astrocytes (ENCLB672PAB; ENCLB174TEA), hippocampus (GSM2322543) and H1 embryonic stem cells (GSM1267196; GSM1267197), as well as aorta (GSM1419084) and lung (GSM2322544; GSM2322545). Virtual 4C plots revealed a clear interaction between the hs966 element and the major promoter of FOXP2 in aorta, neural progenitors (shown in Figure 3) and hippocampus, but not in stem cells or astrocytes from cerebellum.

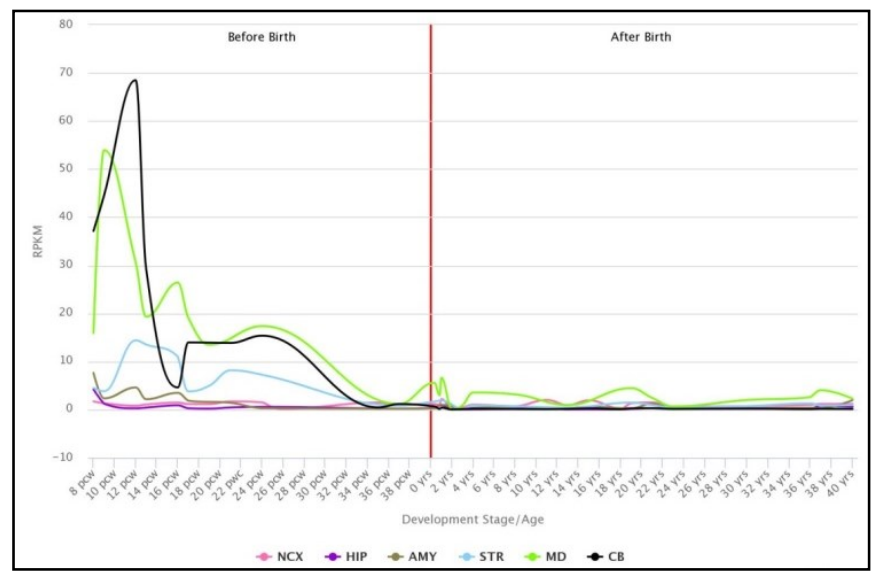

Figure 2. FOXP2 expression during development and postnatal life in six different brain regions according to data from Brainspan. The black line shows the expression profile in cerebellum, which peaks at 12 pcw (post conception week).

Taken together, both the expression profile of FOXP2 during brain development and chromatin interaction data between its promoter and the hs966 Vista element support a role for this enhancer in regulating the expression of this gene in embryonic brain. 
bioRxiv preprint doi: https://doi.org/10.1101/641597; this version posted May 17, 2019. The copyright holder for this preprint (which was not certified by peer review) is the author/funder, who has granted bioRxiv a license to display the preprint in perpetuity. It is made available under aCC-BY-NC-ND 4.0 International license.

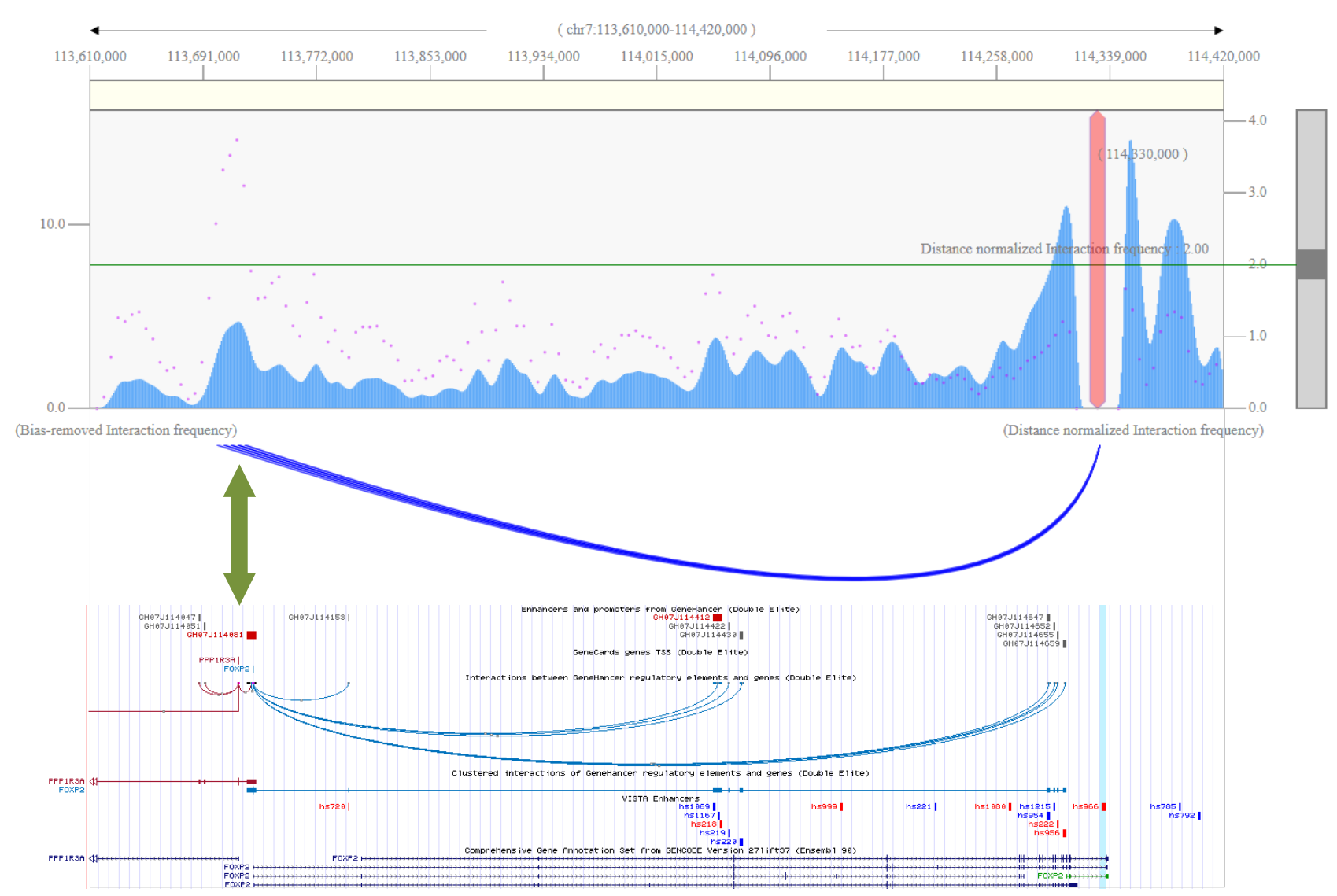

Figure 3. Top: virtual 4C plot showing number of contacts (pink dots) and contacts with distance-normalized interaction frequency $>2.0$ (blue arches) between Vista enhancer hs966 (red vertical bar) and the major promoter of FOXP2 (thick green arrow) in neuronal progenitors using the 3DIV tool. The same region is shown in the UCSC Genome Browser at the bottom, including Gencode annotations, GeneHancer elements and Vista enhancers (hs966 highlighted in blue). Coordinates correspond to the GRCh37 assembly.

Conservation of the hs966 element also supports its functional relevance. As depicted in Figure 4, Multiz alignments in 100 vertebrate genomes reveal very deep conservation. Within this $2.8 \mathrm{~kb}$ element, two smaller blocks show greater constraint with high PhastCons scores. These two regions contain binding sites for several transcription factors in ChIP-seq experiments from neural and non-neural samples (Figure 4, top), as well as a peak of p300 binding in neural cells originated from H1-hESC (ENCSR843ZUP). Both are well conserved in sarcopterygians and actinopyerygians, but not in lamprey.

We then used the sequence of these blocks as query in blastn searches using COGE blast. We found significant hits in the genomes of actinopterygians and in the elephant shark, but not in lamprey or hagfish. Interestingly, alignments were much more significant in spotted gar and elephant shark than in teleostei genomes (which included Danio rerio, Gasterosteus aculeatus, Tetraodon nigroviridis and Takifugu rubripes).
For instance, blastn hits for the left block had $73 \%$ coverage of the query sequence and E-value $=0.0$ in the spotted gar genome, whereas coverage of the query varied between $7.2 \%$ (E-value $=6 \mathrm{e}-04$ ) to $21.2 \%$ (Evalue $=2 \mathrm{e}-21$ ) for Tetraodon and Danio, respectively, and was completely absent in Takifugu. A similar pattern was found for the right block, with very significant hits in Callorhinchus and Lepisosteus (85\% coverage of the query sequence and E-value $=0.0$ in both cases) compared to teleostei (from 23.1\% coverage and Evalue $=8 \mathrm{e}-25$ in Danio to $22.7 \%$ and E-value=2e-33 in Takifugu). In fact, all hits in teleostei genomes were fragmented in two or three blocks.

Of note, the region between these two highly-conserved blocks shows little conservation, particularly a $90 \mathrm{bp}$ segment which seems to be absent even in great ape genomes. Closer inspection of this segment revealed the presence of an (AC) 19 tandem repeat, flanked by two Trich low complexity tracts. This microsatellite is very polymorphic in humans (it spans several SNVs from dbSNP) and is also present in the high-resolution denisovan genome. Primates have a shorter version of 8 repeats, also followed by a poly(T) stretch; however, euarchontoglires, laurasiatheria, and afrotheria have the short version of the dinucleotide repeat without the poly(T) tract, whereas the whole segment is absent in non-eutherian mammals and more basal vertebrates. 
bioRxiv preprint doi: https://doi.org/10.1101/641597; this version posted May 17, 2019. The copyright holder for this preprint (which was

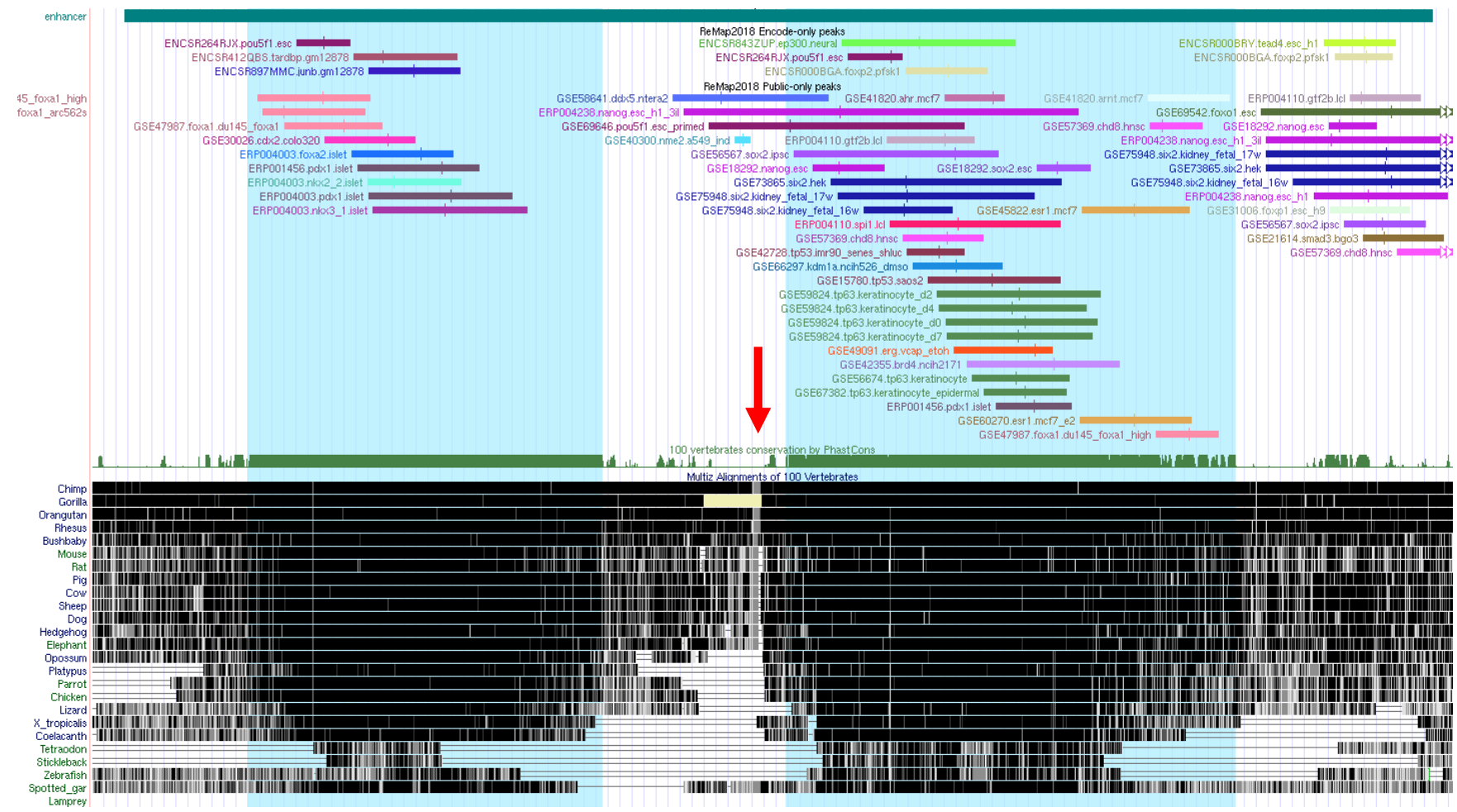

Figure 4. Evolutionary analysis of hs 966 Vista element. Inside this $2.8 \mathrm{~kb}$ element (green rectangle at the top) two smaller regions of great conservation are evident (highlighted in blue), with high PhastCons scores. Conservation in representative vertebrate taxons is shown at the bottom. Transcription factor binding sites from Remap (ENCODE and other publicly available ChIP-seq experiments) are also shown. Both highlighted regions show several TFBS peaks in various cell lines. A region of low conservation (red arrow) includes a microsatellite which is polymorphic in humans but is absent in most vertebrate genomes (see text).

\section{Discussion}

FOXP2 is expressed in mouse and human developing cerebellum and it has been previously implicated in speech and language disorders $(31,32)$. It might be crucial for Purkinje cell development, as it is specifically expressed in those cells, and thus play a relevant role in vocal communication (28-30). Our searches in recent neurodevelopmental expression databases confirmed that FOXP2 expression in developing human brain is highest in cerebellum, around $12 \mathrm{pcw}$. However, there is so far no evidence as to the identity of the cis-regulatory elements responsible for cerebellum-specific expression of this gene.

Our search for Vista enhancers with activity in hindbrain led us to hs966, an enhancer that shows strong and specific enhancer activity in mouse rhombencephalon in embryonic day e11.5, in 6 out of 11 embryos analysed by the Vista enhancer project. This $2.8 \mathrm{~kb}$ element is located in the final intron of FOXP2, suggesting that it might regulate this gene, but experimental evidence for this association was lacking.
In our work we have found that Vista element hs966 displays chromatin marks of active enhancer in human fetal brain samples and in neuronal progenitor cells and that it contacts the major FOXP2 promoter in $\mathrm{H} 1$ derived neuronal progenitor cells. The presence of ChIPseq peaks for several transcription factors in neural and non-neural samples reinforces the role of hs966 as an enhancer. Therefore, we propose that this element is a strong candidate to account for cerebellum-specific expression of FOXP2 during development.

As most Vista elements and developmental enhancers, hs966 is deeply conserved in vertebrate genomes. Two blocks, in particular, are very well conserved in all gnathostome genomes analysed but not in agnatha (lamprey and hagfish). However, conservation of these blocks is weaker in the genomes of teleostei. This suggests that the core elements of the hs966 enhancer were present in the gnathostome ancestor and were conserved in sarcopterygians and non-teleostean actinopterygiians.

Interestingly, these two blocks are separated by a short segment formed by a microsatellite, which is present only in eutherian mammals. Whereas primates and mammals have shorter versions, the microsatellite is expanded in the genomes of anatomically modern humans and denisovans, showing a high degree of polymorphism in repeat number in modern humans. Differences in the size of cerebellum between modern humans and neandertals have been suggested to underlie the cognitive differences that allowed the development of more sophisticated tool usage, language and social skills in modern Homo sapiens (34). It is tempting to speculate that the expansion of this 
bioRxiv preprint doi: https://doi.org/10.1101/641597; this version posted May 17, 2019. The copyright holder for this preprint (which was not certified by peer review) is the author/funder, who has granted bioRxiv a license to display the preprint in perpetuity. It is made available under aCC-BY-NC-ND 4.0 International license.

microsatellite in the middle of hs966 might have optimized the function of this enhancer in humans, and thus increased its regulatory activity on the promoter of FOXP2 during development of the cerebellum.

Several lines of research should be undertaken to confirm our prediction. Deletion of this Vista element in model vertebrates will show whether it plays a significant role on FOXP2 expression in cerebellum at different stages of embryonic development, and any potential structural of functional abnormality derived from its absence. This should be complemented with single-cell transcriptomic analysis of Purkinje and progenitor cells in model vertebrates, as well as chromatin conformation studies aimed at confirming the contact between this enhancer and the promoter of FOXP2 in the same samples.

\section{Acknowledgments}

We would like to thank Dr. José L. Vizmanos for helpful discussions.

\section{References}

1. Butts T, Green MJ, Wingate RJT. Development of the cerebellum: simple steps to make a "little brain." Development. 2014;141:4031-41.

2. Sousa AMM, Meyer KA, Santpere G, Gulden FO, Sestan N Evolution of the Human Nervous System Function, Structure, and Development. Cell. 2017;170:226-47.

3. Harrison PW, Montgomery SH. Genetics of Cerebellar and Neocortical Expansion in Anthropoid Primates: A Comparative Approach. Brain Behav Evol. 2017;89:27485.

4. Carroll SB. Evolution at Two Levels: On Genes and Form. PLoS Biol. 2005;3:e245.

5. Anokhin K, Guijarro P, Yu Q, Jiang S, Efimova O, Oleksiak A, et al. Comprehensive transcriptome analysis of neocortical layers in humans, chimpanzees and macaques. Nat Neurosci. 2017;20:886-95.

6. Ebbert A, Boe A, Potekhina L, Carey A, Bernard A, Arnold J, et al. A comprehensive transcriptional map of primate brain development. Nature. 2016;535:367-75.

7. Somel M, Liu X, Khaitovich P. Human brain evolution: Transcripts, metabolites and their regulators. Nat Rev Neurosci. 2013;14:112-27.

8. Visel A, Minovitsky S, Dubchak I, Pennacchio LA. VISTA Enhancer Browser--a database of tissue-specific human enhancers. Nucleic Acids Res. 2007;35:D88-92.

9. Fishilevich S, Nudel R, Rappaport N, Hadar R, Plaschkes I, Iny Stein T, et al. GeneHancer: genome-wide integration of enhancers and target genes in GeneCards. Database 2017; 10.1093/database/bax028.
10. Zhang Y, An L, Yue F, Hardison RC. Jointly characterizing epigenetic dynamics across multiple human cell types. Nucleic Acids Res. 2016;44:6721-31.

11. Zhang Y, Hardison RC. Accurate and reproducible functional maps in 127 human cell types via 2D genome segmentation. Nucleic Acids Res. 2017 29;45:9823-36.

12. Cheneby J, Gheorghe M, Artufel M, Mathelier A, Ballester, B. ReMap 2018: An updated regulatory regions atlas from an integrative analysis of DNA-binding ChIP-seq experiments. Nucleic Acids Research 2018 46, D1:D267-D275.

13. Lonsdale J, Thomas J, Salvatore M, Phillips R, Lo E, Shad S, et al. The Genotype-Tissue Expression (GTEx) project. Nat Genet. 2013;45:580-5.

14. Miller JA, Ding SL, Sunkin SM, Smith KA, Ng L, Szafer A, et al. Transcriptional landscape of the prenatal human brain. Nature. 2014;508:199-206.

15. Shao Y, Chen C, Shen H, He BZ, Yu D, Jiang S, et al. GenTree, an integrated resource for analyzing the evolution and function of primate-specific coding genes. Genome Res. 2019;29: 682-696.

16. Lindsay SJ, Xu Y, Lisgo SN, Harkin LF, Copp AJ, Gerrelli D, et al. HDBR Expression: A Unique Resource for Global and Individual Gene Expression Studies during Early Human Brain Development. Front Neuroanat. 2016;10:1-6.

17. Petryszak R, Keays M, Tang YA, Fonseca NA, Barrera E, Burdett $\mathrm{T}$, et al. Expression Atlas update-an integrated database of gene and protein expression in humans, animals and plants. Nucleic Acids Res. 2016;44(D1):D74652 .

18. Consortium GTEx. Genetic effects on gene expression across human tissues. Nature. 2017;550:204-13.

19. Landrum MJ, Lee JM, Benson M, Brown G, Chao C, Chitipiralla S, et al. ClinVar: public archive of interpretations of clinically relevant variants. Nucleic Acids Res. 2016;44(D1):D862-8.

20. Hindorff LA, Sethupathy P, Junkins HA, Ramos EM, Mehta JP, Collins FS, et al. Potential etiologic and functional implications of genome-wide association loci for human diseases and traits. Proc Natl Acad Sci U S A. 2009;106:9362-7.

21. Chen EY, Tan CM, Kou Y, Duan Q, Wang Z, Meirelles G, et al. Enrichr: interactive and collaborative HTML5 gene list enrichment analysis tool. BMC Bioinformatics. 2013;14:128.

22. Kuleshov M V., Jones MR, Rouillard AD, Fernandez NF, Duan Q, Wang Z, et al. Enrichr: a comprehensive gene set enrichment analysis web server 2016 update. Nucleic Acids Res. 2016;44(W1):W90-7.

23. Wang, Y, Song, F, Zhang, B, Zhang, L, Xu, J, Kuang, D, Li, D, Choudhary, MNK, Li, Y, Hu, M, et al. The 3D Genome Browser: a web-based browser for visualizing 3D genome organization and long-range chromatin interactions. Genome Biology 2018;19:151.

24. Yang D, Jang I, Choi J, Kim M-S, Lee AJ, Kim H, et al. 3DIV: A 3D-genome Interaction Viewer and database. Nucleic Acids Res. 2018;46(D1):D52-7.

25. Lyons E, Pedersen B, Kane J, Alam M, Ming R, Tang H, et al. 
bioRxiv preprint doi: https://doi.org/10.1101/641597; this version posted May 17, 2019. The copyright holder for this preprint (which was not certified by peer review) is the author/funder, who has granted bioRxiv a license to display the preprint in perpetuity. It is made available under aCC-BY-NC-ND 4.0 International license.

Finding and comparing syntenic regions among Arabidopsis and the outgroups papaya, poplar, and grape: CoGe with rosids. Plant Physiol. 2008;148:1772-81.

26. Lachmann A, Torre D, Keenan AB, Jagodnik KM, Lee HJ, Wang L, Silverstein MC, Ma'ayan A. Massive mining of publicly available RNA-seq data from human and mouse. Nature Communications. 2018;9:1366.

27. Santos A, Tsafou K, Stolte C, Pletscher-Frankild S, O'Donoghue SI, Jensen LJ. Comprehensive comparison of large-scale tissue expression datasets. PeerJ. 2015;3:e1054.

28. Usui N, Co M, Harper M, Rieger MA, Dougherty JD, Konopka G. Sumoylation of FOXP2 Regulates Motor Function and Vocal Communication Through Purkinje Cell Development. Biol Psychiatry. 2017;81:220-230.

29. Fujita-Jimbo E, Momoi T. Specific expression of FOXP2 in cerebellum improves ultrasonic vocalization in heterozygous but not in homozygous Foxp2 (R552H) knock-in pups. Neurosci Lett. 2014;566:162-6.

30. Argyropoulos GPD, Watkins KE, Belton-Pagnamenta E, Liégeois F, Saleem KS, Mishkin M, Vargha-Khadem F. Neocerebellar Crus I Abnormalities Associated with a Speech and Language Disorder Due to a Mutation in FOXP2. Cerebellum. 2018 doi: 10.1007/s12311-018-09893.

31. Nudel R, Newbury DF. Foxp2. Wiley Interdiscip Rev Cogn Sci. 2013;4:547-60

32. Lai CSL, Gerrelli D, Monaco AP, Fisher SE, Copp AJ, FOXP2 expression during brain development coincides with adult sites of pathology in a severe speech and language disorder, Brain 2003:126:2455-2462.

33. Ferland RJ, Cherry TJ, Preware PO, Morrisey EE, Walsh CA Characterization of Foxp2 and Foxp1 mRNA and protein in the developing and mature brain. J Comp Neurol. 2003;460:266-79.

34. Kochiyama T, Ogihara N, Tanabe HC, Kondo O, Amano H, Hasegawa K, et al. Reconstructing the Neanderthal brain using computational anatomy. Sci Rep. 2018;8:6296. 